\title{
Concordancia entre las Evaluaciones de la Articulación Temporomandibular Realizadas con los CDI/TTM y con Imágenes de Resonancia Magnética
}

\author{
Concordance between the Evaluations of the Temporomandibular Joint \\ Performed with the RDC/TMD and with Images of Magnetic Resonance
}

Sonia Osorio*; Elizabeth Peña*; Gloria Baena* \& Adriana Herrera**

OSORIO, S.; PEÑA, E.; BAENA, G. \& HERRERA, A. Concordancia entre las evaluaciones de la articulación temporomandibular realizadas con los CDI/TTM y con imágenes de resonancia magnética. Int. J. Odontostomat., 9(2):177184, 2015.

RESUMEN: Los Criterios Diagnósticos para la Investigación de los Trastornos Temporomandibulares (CDI/TTM) ofrecen un sistema estandarizado para evaluar la Disfunción Temporomandibular (DTM). Sin embargo, la validez del diagnóstico clínico obtenido con estos criterios al compararlo con el diagnóstico obtenido de las imágenes de Resonancia Magnética (RM) es controversial. El objetivo fue determinar la concordancia que existe entre la evaluación clínica realizada con los CDI/TTM e imágenes de RM de la articulación temporomandibular (ATM), de pacientes atendidos en la Clínica Integral del Adulto, de la Escuela de Odontología de la Universidad del Valle, en Cali, Colombia. Se evaluó clínicamente a 36 individuos, con edades comprendidas entre 18 y 60 años, utilizando los CDI/TTM y se les realizaron exámenes de RM. Las variables estudiadas fueron sexo, edad, signos y síntomas de la ATM, Posición Normal del Disco (PND), Desplazamiento Discal con Recaptura (DDCR) y Desplazamiento Discal Sin Recaptura (DDSR). Los datos obtenidos fueron sometidos a pruebas estadísticas para determinar el índice Kappa y características operativas de la prueba clínica. El Índice Kappa fue 0,53, con una concordancia entre las evaluaciones clínicas e imagenológicas moderada. De la población estudiada, 91,6\% fue del sexo femenino, con 31 años como promedio de edad. El signo hallado con mayor frecuencia fue el ruido articular (77\%) y el síntoma más frecuente fue el dolor facial (69\%). La sensibilidad de los CDI/TTM para determinar la PND fue 0,52 y la especificidad 0,87; la sensibilidad para el DDCR fue 0,8780 y la especificidad 0,709; la sensibilidad para el DDSR fue 0,5714 y la especificidad 0,948. La DTM se presentó con mayor frecuencia en las mujeres, con edad promedio de 31 años. Los CDI/TTM pueden considerarse confiables, especialmente para el DDCR; sin embargo, tratamientos invasivos, permanentes o quirúrgicos, requerirían confirmación con un diagnóstico imagenológico para evitar falsos positivos.

PALABRAS CLAVE: articulación temporomandibular, imagen por resonancia magnética, síndrome de la disfunción temporomandibular, disco de la articulación temporomandibular, Criterios Diagnósticos de Investigación en Trastornos Temporomandibulares.

\section{INTRODUCCIÓN}

La disfunción temporomandibular (DTM), referenciada como el dolor orofacial más común (Cao et al., 2009), es una alteración que se caracteriza por sintomatología variada en las regiones craneal, facial y cervical, lo cual genera confusión con el diagnóstico de otras patologías. Estudios epidemiológicos realizados en varios países muestran una alta prevalencia de esta disfunción (Dodic et al., 2006). El National Institute of Dental and Craniofacial Research (2014) reporta que esta condición afecta entre el 5 y el $12 \%$ de la población, con un costo estimado de 4 billones de dólares anuales, siendo clasificada como la condición musculoesquelética más común después del dolor de espalda baja.

En una revisión sistemática realizada por Manfredinni et al. (2011), se evidencia que de las 3.091 personas evaluadas, 1400 (45,3\%) presentaron desórdenes musculares y alteraciones intraarticulares rela-

* Departamento de Morfología, Facultad de Salud, Universidad del Valle, Cali, Colombia.

Subvencionado por: Convocatoria Interna de la Universidad del Valle, año 2013; Código Interno No. 1727. 
cionados con la DTM; a pesar de esta alta prevalencia, aún existe controversia en cuanto a su etiología, diagnóstico y tratamiento. La DTM se divide en alteraciones de la musculatura masticatoria, comúnmente llamada síndrome miofascial, y alteraciones internas de la ATM como desplazamientos discales, inflamaciones y osteoartrosis. Al valorar el funcionamiento de la ATM, los clínicos clasifican el tipo de DTM según criterios diagnósticos universalmente aceptados.

Existen diferentes formatos y guías para evaluar clínicamente la ATM. Sin embargo, se muestran resultados controversiales cuando en investigaciones realizadas para determinar la fiabilidad del diagnóstico clínico, éste se compara con el diagnóstico obtenido de exámenes especializados que valoran objetivamente las alteraciones intrarticulares, tales como la Resonancia Magnética (RM), la cual se considera el patrón de oro o gold standard para identificar alteraciones de la posición discal. En el medio colombiano no es usual requerir exámenes especializados de este tipo debido a los altos costos, por lo cual, el diagnóstico es esencialmente clínico, apoyado en radiografías convencionales que no permiten valorar objetivamente los tejidos blandos.

El presente artículo resume el trabajo de investigación realizado entre los años 2013 y 2014 por la investigadora principal para optar al título de Maestría en Ciencias Biomédicas (Osorio, 2014), el cual tuvo como objetivo primordial determinar la concordancia que existe entre la evaluación clínica de la ATM realizada con los CDI/TTM y la evaluación realizada con imágenes de RM.

\section{MATERIAL Y MÉTODO}

El estudio es de tipo descriptivo, observacional y prospectivo, de pacientes atendidos en la Clínica Integral del Adulto - Clínica de ATM y Dolor Orofacial de la Escuela de Odontología de la Universidad del Valle entre agosto de 2013 y mayo de 2014 . Se seleccionaron 36 teniendo como criterios de inclusión el que tuvieran edades comprendidas entre 18 y 60 años, que aceptaran participar en el estudio y firmaran el Consentimiento Informado, no hubieran sido sometidos a cirugía de la ATM, no tuvieran material de osteosíntesis en la región craneofacial, no estuvieran siendo sometidos a tratamiento de ortodoncia, no tuvieran dispositivos electrónicos como marcapasos, válvulas a nivel cerebral o implantes auditivos, y que no presentaran alteraciones psiquiátricas evidentes o sufrieran de claustrofobia. A los pacientes seleccionados se les aplicaron los CDI/ TTM y en un tiempo máximo de dos semanas después se les tomaron RMs de ambas ATMs en un centro especializado, con un resonador Magnetom Verio PHILIPS de 3.0 teslas, siguiendo como protocolo una secuencia de imágenes sagital boca abierta-boca cerrada, coronal boca cerrada, oblicua boca abierta-boca cerrada, para un total de 9 cortes por paciente, de $3 \mathrm{~mm}$ cada uno, con una distancia entre los cortes de $5 \mathrm{~mm}$.

La lectura de las imágenes de RM fue realizada por una de las co-investigadoras, especialista en Radiología Oral y Maxilofacial, con 10 años de experiencia en este campo, teniendo como guía el formato Research Diagnostic Criteria for Temporomandibular Disoders (RCD/TTD): Development of image analysis criteria and examiner reliability for image analysis (Mansur et al., 2009). Se diseñó un formato para consignar las posiciones del disco articular tomando como criterio diagnóstico la Posición Normal del Disco (PND), el Desplazamiento Discal Con Recaptura (DDCR) y el Desplazamiento Discal Sin Recaptura (DDSR), como se observa en las Figuras 1, 2 y 3, respectivamente. Las pruebas intraobservador realizadas arrojaron un porcentaje de acuerdo del $100 \%$.

La evaluación clínica fue realizada por la investigadora principal, quien se entrenó suficientemente para este fin, luego de un prolongado proceso de estandarización con un experto odontólogo, especialista en Rehabilitación Integral del Adulto, con muchos años de experiencia en la evaluación clínica de la DTM; las pruebas interobsevador realizadas arrojaron un porcentaje de acuerdo del $75 \%$. Para la valoración clínica se utilizó un formato extractado de los Criterios Diagnósticos para la Investigación de los Trastornos Temporomandibulares (CDI/TTM), que tuvo en cuenta las recomendaciones del Consorcio Internacional de Dolor Orofacial (Schiffman et al., 2014), en el cual se incluyeron ítems que pudieran ser comparados con los de la valoración imagenológica aportada por la RM. Las variables estudiadas fueron sexo, edad, síntomas, signos y diagnósticos según la posición del disco articular (PND, DDCR y DDSR). Para mayor información sobre los formatos utilizados para las evaluaciones clínica e imagenológica, consultar a la investigadora principal.

Para analizar los datos y cumplir con los objetivos planteados en el trabajo, se utilizó el Índice Kappa con el fin de determinar el grado de concordancia existente entre el diagnóstico clínico y el diagnóstico imagenológico, y se determinaron las características operativas de la prueba clínica. 


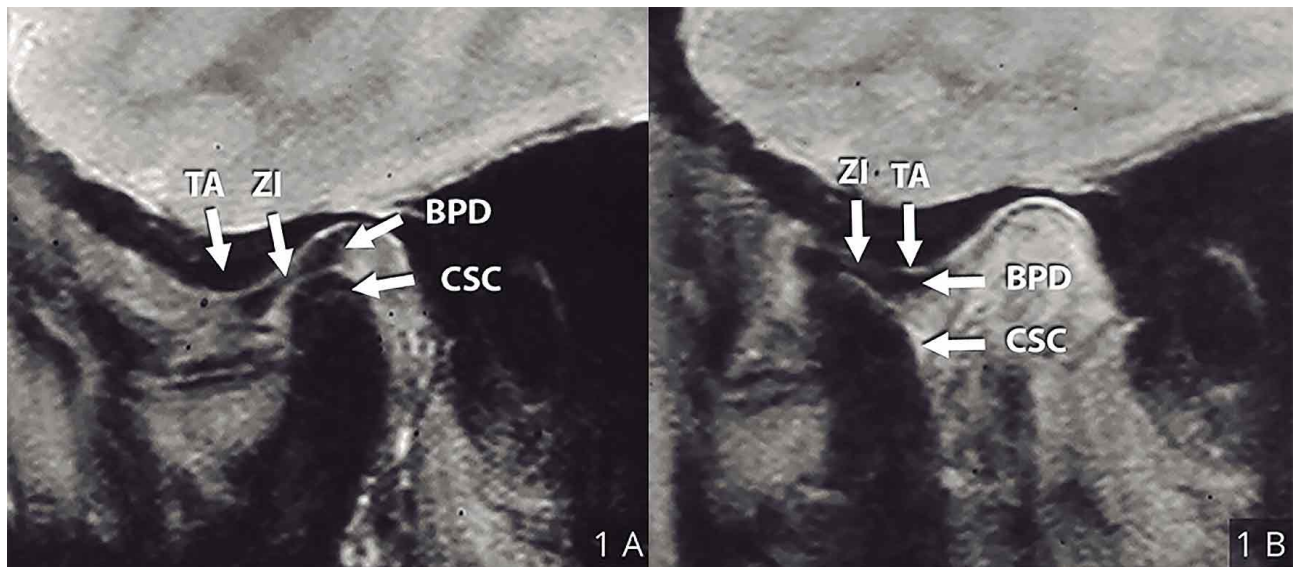

Fig. 1. Imágenes de RM de la ATM con posición normal del disco (PND). A) Posición con boca cerrada. El borde posterior del disco (BPD) se encuentra sobre la cara superior del cóndilo (CSC) y la zona intermedia (ZI) del disco está interpuesta entre el tubérculo articular (TA) y la cara superoanterior del cóndilo. B) Posición con boca abierta. El cóndilo se traslada hacia el tubérculo articular y el disco se encuentra ubicado entre el tubérculo articular y la cara superior del cóndilo.
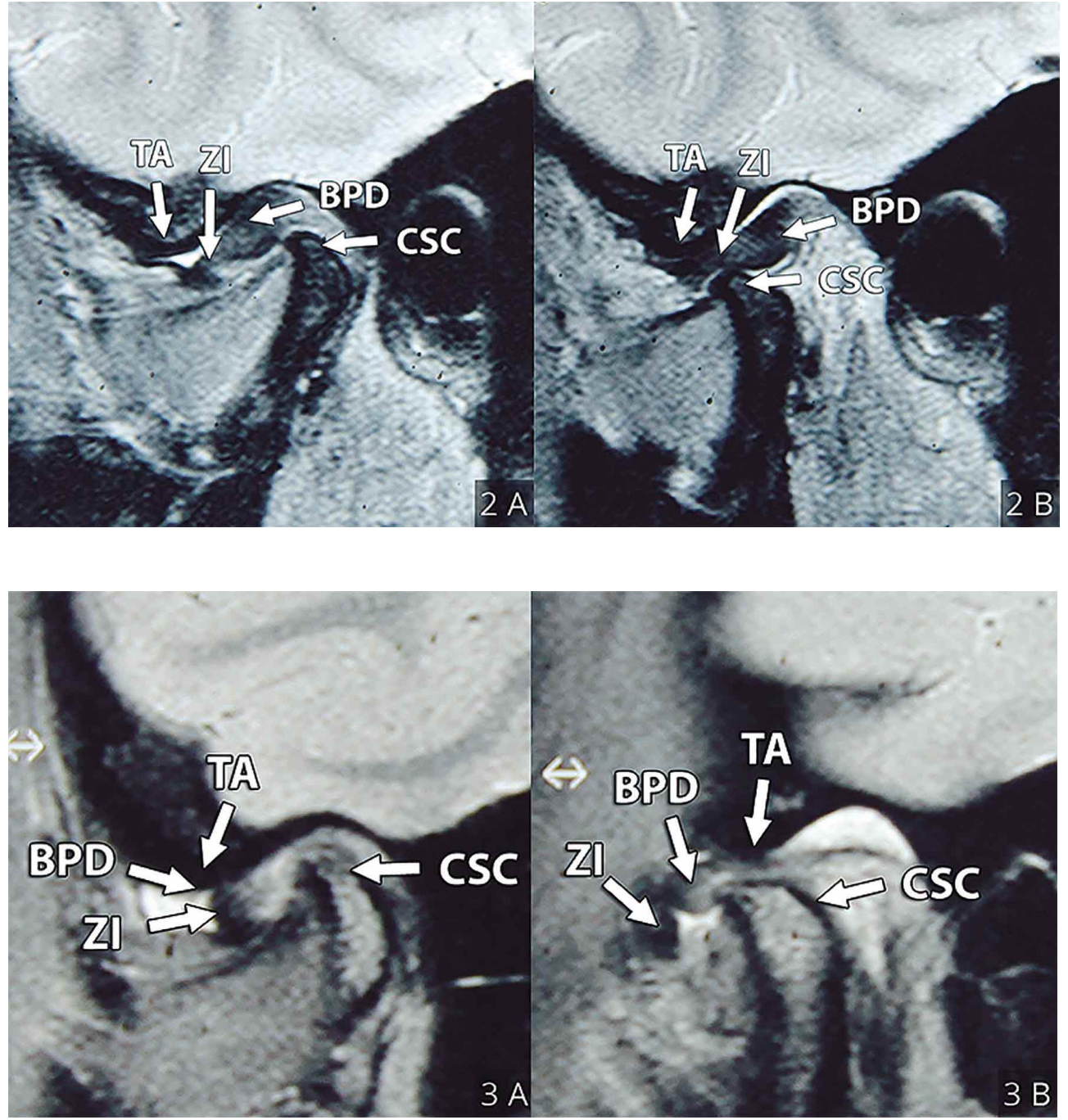

Fig. 2. Imágenes de RM de la ATM con desplazamiento discal con recaptura (DDCR). A) Posición con boca cerrada. El margen posterior del disco (BPD) se encuentra por fuera de la cara superior del cóndilo (CSC). B) Posición con boca abierta. El cóndilo se traslada hacia el tubérculo articular (TA) y el disco se encuentra ubicado entre el tubérculo articular y la cara superior del cóndilo.

Fig. 3. Imágenes de RM de la ATM con desplazamiento discal sin recaptura (DDSR). A) Posición con boca cerrada. El borde posterior del disco (BPD) está ubicado por delante con respecto a la cara superior del cóndilo (CSC). B) Posición con boca abierta. El cóndilo se traslada hacia el tubérculo articular (TA), pero el disco continúa ubicado anteriormente con respecto al cóndilo, convirtiéndose en un obstáculo para la apertura oral. 


\section{RESULTADOS}

De los 36 pacientes estudiados, 3 eran de sexo masculino $(8,3 \%)$ y 33 de sexo femenino $(91,6 \%)$. Las edades de las mujeres fueron más diversas y menores, con un límite inferior de 18 y uno superior de 60 años, para una mediana de 31 , en tanto que para los hombres el límite inferior fue de 32 y el superior de 58 años, para una mediana de 39.

Con respecto a la prevalencia de los síntomas estudiados y el promedio de edad hallado para su ocurrencia, se encontraron los siguientes resultados: ruido articular ( $77 \%$ y 35 años), dolor facial (69\% y 34 años), cervicalgia (55\% y 37 años), bloqueos (50\% y 33 años), cefalea ( $47 \%$ y 37 años) y sintomatología del oído como acúfenos y otalgia (44\% y 40 años).
El índice Kappa hallado para la concordancia entre la evaluación clínica y la evaluación de las imágenes de RM para los tres diagnósticos de posición del disco articular fue 0,53, con Límite inferior de 0,36 y Límite superior de 0,70 , lo que representa una concordancia moderada.

A partir de los diagnósticos clínico e imagenológico de cada paciente para la posición del disco articular, se determinaron las características operativas de los CDI/TTM, tales como sensibilidad, especificidad, proporción de falsos positivos (PFP), proporción de falsos negativos (PFN), el valor predictivo positivo (VPP) y el valor predictivo negativo (VPN); los resultados obtenidos para cada una de las posiciones del disco están representados en las Tablas I, II y III.

Tabla I. Características operativas de la evaluación clínica comparada con la evaluación imagenológica para el diagnóstico de PND.

\begin{tabular}{lccc}
\hline & & \multicolumn{2}{c}{ Intervalo de Confianza del 95\% } \\
\cline { 3 - 4 } Prueba PND & Estimación puntual & Límite inferior & Límite superior \\
\hline Sensibilidad & 0,5294 & 0,41 & 0,64 \\
Especificidad & 0,8727 & 0,80 & 0,95 \\
PFP & 0,1272 & 0,05 & 0,20 \\
PFN & 0,4705 & 0,36 & 0,59 \\
VPP & 0,5625 & 0,45 & 0,68 \\
VPN & 0,8571 & 0,78 & 0,94 \\
\hline
\end{tabular}

Tabla II. Características operativas de la evaluación clínica comparada con la evaluación imagenológica para el diagnóstico de DDCR.

\begin{tabular}{lccc}
\hline & & \multicolumn{2}{c}{ Intervalo de Confianza del 95\% } \\
\cline { 3 - 4 } Prueba DDCR & Estimación puntual & Límite inferior & Límite superior \\
\hline Sensibilidad & 0,878 & 0,80 & 0,95 \\
Especificidad & 0,7096 & 0,60 & 0,81 \\
PFP & 0,2903 & 0,19 & 0,40 \\
PFN & 0,1219 & 0,05 & 0,20 \\
VPP & 0,8 & 0,71 & 0,89 \\
VPN & 0,878 & 0,80 & 0,95 \\
\hline
\end{tabular}

Tabla III. Características operativas de la evaluación clínica comparada con la evaluación imagenológica para el diagnóstico de DDSR.

\begin{tabular}{lccc} 
& & \multicolumn{2}{c}{ Intervalo de Confianza del $\mathbf{9 5 \%}$} \\
\cline { 3 - 4 } Prueba DDSR & Estimación puntual & Límite inferior & Límite superior \\
\hline Sensibilidad & 0,5714 & 0,46 & 0,69 \\
Especificidad & 0,9482 & 0,90 & 1,00 \\
PFP & 0,051 & 0,00 & 0,10 \\
PFN & 0,4285 & 0,31 & 0,54 \\
VPP & 0,72 & 0,62 & 0,82 \\
VPN & 0,57 & 0,46 & 0,68 \\
\hline
\end{tabular}

PFP= Proporción de Falsos Positivos; PFN= Proporción de Falsos Negativos; VPP= Valor Predictivo Positivo; VPN= Valor Predictivo Negativo. 
El área bajo la curva ROC es un indicador global de la precisión de los CDI/TTM y representa gráficamente la razón de verdaderos positivos frente a la razón de falsos positivos. Se encontró que el área bajo la curva para el diagnóstico de PND fue 0,701, para el de DDCR 0,794 y para el de DDSR 0,76, ubicándose todos por encima de la línea de no discriminación.

\section{DISCUSIÓN}

El porcentaje de usuarios que consulta por DTM en la Clínica Integral del Adulto de la Universidad del Valle es, en su mayoría, del sexo femenino, lo cual corrobora lo reportado en otros estudios con respecto a que este trastorno es 1,5 a 4 veces más frecuente en mujeres que en hombres (Kitsoulis et al., 2011).

El rango de 32 a 37 años con mediana de 31 años para la aparición de la sintomatología dolorosa relacionada con la DTM se encuentra por debajo del reportado en otros estudios como el de Yekkalam \& Wanman (2014), quienes la reportaron en mujeres entre 35 y 50 años.

El signo más prevalente de la DTM encontrado, el ruido articular, seguido del síntoma de dolor facial, es un resultado similar al encontrado por autores como Pedroni et al. (2003). El click es considerado como un criterio diagnóstico para indicar una alteración en el funcionamiento normal del complejo cóndilo disco de la ATM y se ha sugerido que existe una relación directa entre el dolor de la ATM y los desplazamientos discales (Fujiwara et al., 2013). El síntoma de menor prevalencia fue la sintomatología en el oído, que incluyó la otalgia y el tinnitus. El $44 \%$ encontrado en el sexo femenino es similar a lo encontrado por Shanaz \& Mustafa (2014) al evaluar 203 pacientes con DTM. No se comprende bien la relación entre los síntomas del oído y la DTM, pero se cree que existe una relación anatómica y funcional entre la ATM y el oído medio. Al respecto, Ramírez et al. (2009), encontraron un ligamento llamado "Disco maleolar" que une la zona retrodiscal de la ATM con la cabeza del martillo del oído medio.

El Índice Kappa de 0,53 (moderado) hallado para la concordancia entre las evaluaciones clínica e imagenológica de los diagnósticos de PND, DDCR y DDSR, evidencia que la sola evaluación clínica podría ser utilizada para emitir un diagnóstico acertado. Dado que el intervalo de confianza fue del $95 \%$ y que esta estimación podría variar entre $36 \%$ y $70 \%$, la concordancia podría alcanzar un nivel bueno. Los reportes encontrados sobre el grado de acuerdo existente entre los diagnósticos clínico e imagenológico de las alteraciones del complejo cóndilo disco de la ATM son controversiales, pues en tanto algunos autores (Emshoff et al., 2002; Barclay et al., 1999; Park et al., 2012) reportan un grado de acuerdo leve y bajo entre ambos diagnósticos, concluyendo que los CDI/TTM tienen un valor limitado para detectar la posición discal y que es necesario complementar el diagnóstico clínico con imágenes de RM, otros (Manfredinni \& Nardini, 2008; Kannan \& Sathasivasubramanian, 2011) reportan un nivel de concordancia bueno, reconociendo que con una historia clínica completa es posible detectar alteraciones del complejo cóndilo disco de forma acertada, sin desconocer la importancia que tienen los exámenes especializados.

En cuanto a las características operativas de la evaluación clínica para determinar el rendimiento de los CDI/TTM, la sensibilidad hallada para la PND fue 0,52 , más alta que la reportada por Emshoff et al. de 0,25 , a diferencia de la especificidad, la cual se encontró en un valor similar $(0,87$ y 0,85 , respectivamente). Estos resultados indican que los CDI/TTM son más específicos que sensibles para el diagnóstico de la PND, por lo que la prueba clínica en la mitad de los casos daría un diagnóstico de normalidad cuando en realidad existe DTM, pero es más eficaz para diagnosticar la disfuncionalidad.

Los resultados para el diagnóstico de DDCR muestran una sensibilidad de 0,87 , que concuerda con la reportada en otras investigaciones como la de Barclay et al. de 0,78, Emshoff et al. de 0,85 y la de Galhardo et al. (2013) de 0,83, lo que indica que la prueba clínica es eficaz para detectar este tipo de disfunción de la ATM.

En cuanto a la especificidad para el DDCR, el resultado de la presente investigación $(0,70)$ se aproxima a la reportada por Yatani et al. (1998), solamente para el signo de Click $(0,89)$, lo que indica que la prueba puede identificar verdaderos negativos en un gran porcentaje. Por el contrario, Emshoff et al. reportan una especificidad muy baja para este diagnóstico $(0,21)$, lo que se presta a reportar muchos falsos negativos, por lo que concluyen que el examen clínico es insuficiente para valorar este tipo de disfunción.

Para el tercer diagnóstico (DDSR), los resultados para la sensibilidad $(0,57)$ y la especificidad $(0,94)$ 
indican que la prueba clínica (CDI/TTM) se presta para diagnosticar este trastorno solo en la mitad de los casos en que los individuos padecen la disfunción, y que en un alto porcentaje los individuos a los cuales no se les diagnosticó el trastorno realmente no lo tenían, es decir, que la prueba no es muy eficaz para detectar verdaderos positivos y sí lo es para detectar verdaderos negativos. Con respecto al resultado para la sensibilidad de solo el $57 \%$, esto podría explicarse por el hecho de que muchos pacientes quienes sufren de esta disfunción en forma crónica resultan asintomáticos y en el estudio se encontraron pacientes quienes presentaban DDSR de la ATM pero que eran totalmente asintomáticos $\mathrm{y}$, de igual manera, no refirieron antecedentes de limitación en la funcionalidad. En estos casos, resulta difícil poder detectar clínicamente alguna alteración que sí la detecta el Gold Standard o imagen de RM, pues al parecer en algunos pacientes la ATM se adapta biomecánicamente al desplazamiento discal y no limita ninguna de las funciones básicas de esta articulación. Al respecto, Yatani et al., informaron en su estudio que la prevalencia del desplazamiento discal en asintomáticos fue del 16 al 33\%. Estos resultados difieren de los reportados por Üsümez et al. (2004), quienes encontraron un porcentaje de acuerdo entre la evaluación clínica y la imagenológica para el DDSR del $81 \%$, recomendando la sola examinación clínica como un método exacto para detectar esta disfunción.

La especificidad de los CDI/TTM para el DDSR fue del $94 \%$, siendo éste el valor más alto de los encontrados para los tres diagnósticos; lo anterior indica que la prueba es muy eficaz para diagnosticar esta alteración y permite identificar con precisión los verdaderos negativos y detecta pocos falsos positivos.

Los valores obtenidos para las áreas bajo la curva ROC indican que los CDI/TTM constituyen una prueba clínica buena para diagnosticar los desplazamientos discales del complejo cóndilo disco de la ATM, pero son regulares para identificar la posición normal del disco. En contraste, Galhardo et al., reportaron que la prueba clínica es regular para identificar las alteraciones de la ATM.

El examen clínico para detectar alteraciones intraarticulares de la ATM ha sido cuestionado y comparado con exámenes especializados como la RM. Barclay et al., concluyen que los CDI/TTM diagnostican desarreglos internos de la ATM, pero que la sola examinación clínica tiene un valor limitado para detectar la posición discal. De igual manera, Galhardo et al. consideran que la evaluación clínica presenta un alto número de falsos positivos, lo que podría limitar su uso como herramienta diagnóstica. Por el contrario, Donlon \& Moor (1987) compararon la examinación clínica, la RM y la artrotomografía con los hallazgos quirúrgicos en casos de DTM, y reportaron que la mejor correlación fue la de la evaluación clínica. En este sentido, Yatani et al. \& Üsümez et al. consideran que los desplazamientos discales de la ATM pueden ser diagnosticados con exactitud con la sola examinación clínica, mientras que para Kannan \& Sathasivasubramanian, el diagnóstico clínico, conjuntamente con una anamnesis adecuada y una exploración física completa, es confiable y apropiado, aunque la RM juegue un papel importante en el diagnóstico.

Los resultados de este estudio permiten reconocer que los CDI/TTM constituyen un instrumento que está bien formulado, que evalúa signos y síntomas relacionados con alteraciones en la posición del complejo cóndilo - disco de la ATM y que presenta una alta sensibilidad y especificidad para el DDCR. Sin embargo, la diferencia existente entre este diagnóstico clínico y el Gold Standard podría explicarse porque existen pacientes quienes presentan desplazamiento discal de la ATM sin presentar síntomas y signos clínicos (Üsümez et al.). Otra explicación podría ser el tiempo que puede transcurrir entre la examinación clínica y la toma de la $\mathrm{RM}$, generalmente dos semanas, que podrían prestarse a cambios en la posición discal o en la función.

\section{CONCLUSIONES}

Dado que se encontró una concordancia moderada entre las evaluaciones clínica e imagenológica para los tres diagnósticos de posición del disco estudiadas, los CDI/TTM pueden ser considerados como una herramienta aceptable para llegar a un diagnóstico acertado de la DTM, pero no es determinante.

Por la alta sensibilidad y mediana especificidad obtenidas para el diagnóstico clínico de DDCR, los CDI/ TTM pueden considerarse confiables; sin embargo, para tratamientos invasivos, permanentes o quirúrgicos, se requeriría de confirmación con el diagnóstico imagenológico.

En aquellos diagnósticos de DTM donde la prueba clínica presenta una sensibilidad cercana del $50 \%$, pero una alta especificidad, se requeriría también del diagnóstico imagenológico para evitar futuras compli- 
caciones para el paciente. En estos casos, la prueba clínica no es concluyente y se requeriría mayor inves- tigación al respecto, dado que el profesional clínico basa la intervención del paciente en este resultado.

OSORIO, S.; PEÑA, E.; BAENA, G. \& HERRERA, A. Concordance between the evaluations of the temporomandibular joint performed with the RDC/TMD and with images of magnetic resonance. Int. J. Odontostomat., 9(2):177-184, 2015.

ABSTRACT: The Research Diagnostic Criteria for Temporomandibular Disorders (RDC/TMD) offer a standardized system to clinically evaluate the most common subtypes of Temporomandibular Disorders (TMD). However, the validity of the clinical diagnose obtained with these criteria when compared with the diagnose obtained from the images of Magnetic Resonance (MR) is controversial. The objective of this study was to determine the concordance existing between the clinical evaluation obtained from the RDC/TMD and that obtained from MR images of the temporomandibular joint (TMJ) of patients attending the Clínica Integral del Adulto - Clínica de ATM, of the Escuela de Odontología of the Universidad del Valle, Cali, Colombia. A population of 36 patients of both sexes, with ages between 18 and 60 years, were clinically evaluated with the RDC/TMD and MR. The variables considered were sex, age, signs and symptoms of the TMJ, Normal Disc Position (NDP), Disc Displacement with Reduction (DD-R) and Disc Displacement without Reduction (DD-NR). The data obtained were submitted to statistical tests to determine the Kappa Index and operative characteristics of the clinical evaluation. The Kappa Index obtained was 0.53 , which shows that the concordance between the clinical and the imagenological evaluations is moderate. Of the total population studied, $91.6 \%$ were females, with 31 years as average age. The most frequent sign found was the joint sound (77\%) and the most frequent symptom was facial pain $(69 \%)$. The sensitivity of the RDC/TMD to determine NDP was 0.52 and the specificity 0.87 ; the sensitivity for the DD-R was 0.8780 and the specificity 0.709 ; the sensitivity for the DD-NR was 0.5714 and the specificity 0.948 . The TMD were found most frequent in women, with average age of 31 years. The RDC/TMD can be considered reliable, especially for the DD-R; however, invasive, permanent or surgical treatments would require confirmation with imagenological diagnoses to avoid false positives.

KEY WORDS: temporomandibular joint, magnetic resonance images, temporomandibular disorder syndrome, temporomandibular joint disc, Research Diagnostic Criteria for Temporomandibular Disorders.

\section{REFERENCIAS BIBLIOGRÁFICAS}

Barclay, P.; Hollender, L. G.; Maravilla, K. R. \& Truelove, E. L. Comparison of clinical and magnetic resonance imaging diagnosis in patients with disk displacement in the temporomandibular joint. Oral Surg. Oral Med. Oral Pathol. Oral Radiol. Endod., 88(1):37-43, 1999.

Cao, Y.; Xie, Q. F.; Li, K.; Light, A. R. \& Fu, K. Y. Experimental oclussal interference induces long-term masticatory muscle hyperalgesia in rats. Pain, 144(3):287-93, 2009.

Dodic, S.; Stanisic-Sinobad, D.; Vukadinovic, M.; Millic, A. \& Sinobad, V. The prevalence of craniomandibular disorders in the military population of the Republic of Serbia. Med Pregl., 59(5-6):259-64, 2006.

Donlon, W. C. \& Moor K. L. Comparison of magnetic resonance imagining, arthrotomography and clinical and surgical findings in temporomandibular joint internal derangements. Oral Surg. Oral Med. Oral Pathol., 64(1):2-5, 1987.

Emshoff, R.; Brandlmaier, I.; Bösch, R.; Gerhard, S.; Rudisch, A. \& Bertram, S. Validation of the clinical diagnostic criteria for temporomandibular disorders for the diagnostic subgroup - disc derangement with reduction. J. Oral Rehabil., 29(12):1139-45, 2002.
Fujiwara, M.; Honda, K.; Hasegawa, Y. \& Urade, M. Comparison of joint in pain in patients diagnosed with and without articular disc displacement without reduction based on the Research Diagnostic Criteria for Temporomandibular Disorders. Oral Surg. Oral Med. Oral Pathol. Oral Radiol., 116(1):9-15, 2013.

Galhardo, A. P. ; Da Costa, L. C.; Gebrim, E. M.; Gomez, R. L.; Mukai, M. K.; Yamaguchi, C. A.; Bernardo, W. M.; Soares, J. M.; Baracat, E. C. \& Gil, C. The Correlation of research diagnostic criteria for temporomandibular disorders and magnetic resonance imaging: a study of diagnostic accuracy. Oral Surg. Oral Med. Oral Pathol. Oral Radiol, 115(2):277-84, 2013.

Kannan, A. \& Sathasivasubramanian, S. Comparative study of clinical and magnetic resonance imaging diagnosis in patients with internal derangement of the temporomandibular joint. J. Indian Acad. Oral Med. Radiol., 23(4):569-75, 2011.

Kitsoulis, P.; Marini, A.; LLiou, K.; Galani, V.; Zimpis, A.; Kanavaros, P. \& Paraskevas, G. Signs and symptoms of temporomandibular joint disorders related to the degree of mouth opening and hearing loss. BioMed Central, 11(5):1-8, 2011. 
Mansur, A.; Hollender, L. \& Schiffman, E. Research diagnostic Criteria for temporomandibular disoders (RCD/TTD): Development of image analysis criteria and examiner reliability for image analysis. Oral Surg. Oral Med. Oral Pathol. Oral Radiol. Endod., 107(6):844-60, 2009.

Manfredini, D. \& Nardini, G. Agreement between Research Diagnostic Criteria for Temporomandibular and magnetic resonance diagnoses of temporomandibular disc displacement in a patient population. Int. J. Oral Maxilofac. Surg., 37(7):612-6, 2008.

Manfredinni, D.; Guarda-Nardini, L.; Winocur, E.; Piccotti, F.; Ahlberg, J. \& Lobbezoo, F. Research diagnostic criteria for temporomandibular disorders: a systematic review of axis I epidemiologic findings. Oral Surg. Oral Med. Oral Pathol. Oral Radiol. Endod., 112(4):453-62, 2011.

National Institute of Dental and Craniofacial Research. Prevalence of TMDJ and its Signs and symptoms. 2014. Disponible en: http://www.nidcr.nih.gov/DataStatistics/ FindDataByTopic/FacialPain/PrevalenceTMJD.htm. 2014.

Osorio, S. Concordancia entre la evaluación clínica de la articulación temporomandibular mediante el formato CDI/ TTM e imágenes de resonancia magnética nuclear. Trabajo de investigación para Maestría en Ciencias Biomédicas. Universidad del Valle, Cali, Colombia, 2014.

Park, J. W.; Song, H. H.; Roh, H.S.; Kim, Y. K. \& Lee, J. Y. Correlation between clinical diagnosis based on RDC/ TMD and MRI findings of TMJ internal derangement. Int. J. Maxillofac Surg., 41(1):103-8, 2012.

Pedroni, C. R.; De Oliveira, A. S. \& Guaratini, M. I. Prevalence study of signs and symptoms of temporomandibular disorders in university students. J. Oral Rehabil., 30(3):283-9, 2003.

Ramirez, A. L. M.; Ballesteros, A. L. E. \& Sandoval, O. G. P. A direct anatomical study of the morphology and functionality of disco-malleolar and anterior malleolar ligaments. Int. J. Morphol., 27(2):367-79, 2009.

Shanaz, M. G. \& Mustafa, J. A. Retrospective Study of a series of 203 patients with temporomandibular joint disorders presenting at school of dentistry, University of Sulaimani. EJS, 10(9):216-25, 2014.

Schiffman, E.; Ohrbach, R.; Truelove, E.; Look, J.; Anderson, G.; Goulet, J. P.; List, T.; Svensson, P.; Gonzalez, Y.; Lobbezoo, F.; Michelotti, A.; Brooks S. L.; Ceusters, W.; Drangsholt, M.; Ettlin, D.; Gaul, C.; Goldberd, L. J.; Haythornthwaite, J. A.; Hollender, L.; Jensen, R.; John, M. T.; De Laat, A.; De Leeuw, R.; Smith, B.; Visscher, C. H.; Zakrzewska, J. \& Dworkin, S. F. Diagnostic criteria for temporomandibular disorders (DC/TMD) for clinical and research applications: recommendations of the international RDC/TMD Consortium Network and Orofacial Pain Special Interest Group. J. Oral Facial Pain Headache, 28(1):6-27, 2014.

Üsümez, S.; Öz, F. \& Guray, E. Comparison of clinical and magnetic resonance imaging diagnoses in patients with TMD history. J. Oral Rehabil., 31(1):52-6, 2004.

Yatani, H.; Sonoyama, W.; Kuboki, T.; Matsuka, Y.; Orsini, M. \& Yamashita, A. The validity of clinical examination for diagnosing anterior disk displacement with reduction. Oral Surg. Oral Med. Oral Pathol. Oral Radiol. Endod., 85(6):647-53, 1998.

Yekkalam, N. \& Wanman, A. Prevalence of signs and symptoms indicative of temporomandibular disorders and headaches in 35-, 50-, 75- year-olds living in Västerbotten, Sweden. Acta Odontol. Scand., 72(6):45865, 2014.

Dirección para Correspondencia:

Gloria Baena

Departamento de Morfología

Facultad de Salud

Universidad del Valle

Cali

COLOMBIA

Tel: (572) 5185627.

Email: gloria.baena@correounivalle.edu.co

Recibido : 06-01-2015

Aceptado: 02-06-2015 